Acta Crystallographica Section A

Foundations of

Crystallography

ISSN 0108-7673

Editor: D. Schwarzenbach

\title{
Anomalous scattering method in crystallography on the basis of parametric X-radiation
}

\section{D. Feranchuk and A. Ulyanenkov}

Copyright (C) International Union of Crystallography

Author(s) of this paper may load this reprint on their own web site provided that this cover page is retained. Republication of this article or its storage in electronic databases or the like is not permitted without prior permission in writing from the IUCr. 
Acta Crystallographica Section A

Foundations of

Crystallography

ISSN 0108-7673

Received 23 July 2004

Accepted 11 October 2004

(C) 2005 International Union of Crystallography

Printed in Great Britain - all rights reserved

\section{Anomalous scattering method in crystallography on the basis of parametric $X$-radiation}

\author{
I. D. Feranchuk ${ }^{\mathrm{a}}$ and A. Ulyanenkov ${ }^{\mathrm{b} *}$
}

${ }^{\mathbf{a}}$ Byelorussian State University, F. Skariny Av., 4, 220050 Minsk, Republic of Belarus, and ${ }^{\mathbf{b}}$ Bruker AXS, Östl. Rheinbrückenstrasse 50, 76187 Karlsruhe, Germany. Correspondence e-mail: fer@open.by

Spectra of parametric X-radiation (PXR) in the range of anomalous dispersion of atoms of a crystallographic unit cell are theoretically analyzed. Characteristics of PXR are calculated for both ultrarelativistic $(E \geq 50 \mathrm{MeV})$ and nonrelativistic ( $E \simeq 100 \mathrm{keV}$ ) electrons interacting with complex organic crystals. The analysis of the PXR angular distribution is shown to permit the realization of the anomalous scattering method for the direct measurement of structure amplitude phases.

\section{Introduction}

Parametric X-radiation (PXR) from the electrons in a crystal was theoretically predicted by Baryshevsky \& Feranchuk (1972), Garibyan \& Yang (1972) and Ter-Mikaelyan (1972). At present, it is widely investigated by many experimental and theoretical groups (see, for example, Backe et al., 2001). In these investigations, the main attention is devoted to the possibility of PXR application as the source of quasi-monochromatic X-rays (Rullhansen et al., 1998). However, the PXR spectra also deliver important information about the structure of crystals where the radiation is generated. The analysis of these spectra, as shown first by Feranchuk (1979), can essentially complete the standard methods based on the conventional diffraction of external X-rays within the investigated crystals. The purpose of the present work is to justify one more possible PXR application, namely, for the direct measurement of the phases of structure amplitudes, which are required for unambiguous evaluation of the electron density in X-ray structure analysis.

Development of various physical methods for the direct measurement of the phases of structure amplitudes is of great interest in X-ray diffractometry (Woolfson \& Fan, 1995). These methods are especially valued when the structure of organic crystals with a complicated multiple-atom elementary unit cell has to be determined. Several simple and effective methods for solution of the phase problem were suggested a long time ago for special cases (Vainshtein, 1978). The first is the method of isomorphic replacement when the heavy atom is introduced into the investigated structure (Harker, 1956). The second approach is the anomalous scattering method (ASM), when intensities of the reflected waves are measured for two different X-ray wavelengths close to the absorption edge of one of the atoms in the crystallographic unit cell (Bijvoet, 1954). Both methods permit the phase information to be obtained even in the case of rather thin or mosaic crystals, when the kinematic theory of diffraction can be applied. For practical X-ray structure analysis, it is also essential that the phases can be calculated through the solutions of simple algebraic equations (Porai-Koshitz, 1968; Attfield, 1990; Hendrickson, 1991).

Several methods for the direct measurement of the phases have been suggested on the basis of the interference phenomena in connection with various multiple-beam diffraction set-ups. This approach is actively developed by many authors (for example, Hart \& Lang, 1961; Colella, 1974; Post, 1977; Chang, 1984; Shen \& Colella, 1987; Weckert \& Hümmer, 1997; Shen \& Huang, 2001; Stetsko et al., 2001; and references therein). As a rule, the interference between the waves diffracted by different planes is essential for rather thick and perfect crystals when effects of dynamical X-ray diffraction are strong enough. However, the realization of such methods for macromolecular organic crystals is very problematical as most of them are thin and mosaic. Besides, the evaluation of the phases in the scope of multiple-beam diffraction even for ideal crystals requires rather complicated and precise measurements.

The further development of simple and universal realizations of the ASM is still attractive due to its kinematical domain. There are two known problems in the universal application of the ASM: (i) an X-ray source of monochromatic radiation with smoothly varied wavelength has to be used and (ii) absorption edges in most organic crystals correspond to soft X-rays (for example, the heaviest atom in amino acids, sulfur, has a $K \alpha$ line corresponding to the photon energy $\hbar \omega_{K} \simeq 2.45 \mathrm{keV}$, or the wavelength $\lambda_{K} \simeq 5.019 \AA$ ). Both problems occur for ASM application under laboratory conditions because of the absence of tunable soft X-ray sources. They can be overcome with the use of synchrotron radiation; however, there are also some difficulties in this case, i.e. standard crystal monochromators are not efficient for soft X-rays (Hart, 1990).

The proposed ASM realization in the present paper is based on the analysis of the spectra of the parametric X-radiation 
generated by the electrons passing through the investigated crystal. This approach could be realized under laboratory conditions with PXR from ultrarelativistic electrons (with energy $E \sim 30-50 \mathrm{MeV}$ ), when the crystal is placed inside the compact betatron (Kaplin et al., 2002). This set-up is considered in $\$ 2$, and $\S 3$ deals with application of PXR from nonrelativistic electrons (energy $E \sim 100 \mathrm{keV}$ ), which have been investigated by Feranchuk \& Ulyanenkov (1999, $2001 a, b)$ and Feranchuk et al. (2000). In $\S 4$, the phase of a structure amplitude is calculated on the basis of the PXR spectrum simulated for the organic crystal $\mathrm{C}_{13} \mathrm{H}_{14} \mathrm{~N}_{2} \mathrm{OS}$. The conditions at which the electron beam yielding PXR does not destroy the investigated crystal are also discussed.

\section{Anomalous scattering method with the spectrum of PXR from ultrarelativistic electrons}

A detailed description of the interaction between the ultrarelativistic electrons and crystals is presented by Baryshevsky \& Feranchuk (1983) and Feranchuk \& Ivashin (1985), and here we briefly give the results of these papers. For a crystal that is arbitrarily orientated to the velocity $\mathbf{v}$ of an incident electron with energy $E \gg m c^{2}$, where $m$ is the electron mass and $c$ is the velocity of light (Fig. 1), the electromagnetic field of the particle can be represented as the beam of pseudophotons. This beam has similar properties to the beam of real photons with the wavevectors $\mathbf{k} \simeq \omega \mathbf{v} / c^{2}$ concentrated in the narrow cone directed along the vector $\mathbf{v}$ and with divergence angle $\Delta \theta \simeq m c^{2} / E$. The density of the pseudo-photon frequency distribution is defined by the following formula (Baryshevsky \&Feranchuk, 1983):

$$
n(\omega) \mathrm{d} \omega \simeq \frac{\alpha}{\pi \omega} \ln \left(\eta \frac{E}{\hbar \omega}\right) \mathrm{d} \omega .
$$

Here $\alpha=e^{2} / \hbar c \simeq 1 / 137$ is the fine structure constant and $\eta$ is a value close to unity. The kinematics of the electromagnetic interaction between the electron and the crystal is equivalent

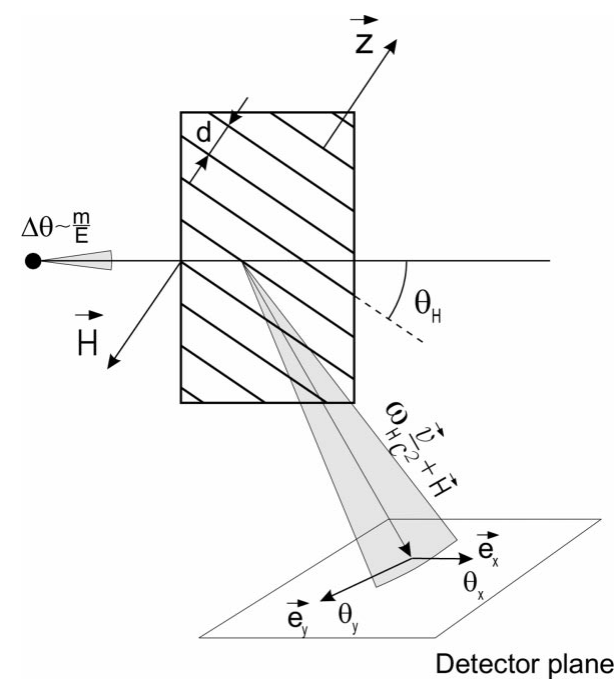

Figure 1

Sketch of the PXR experiment in the case of relativistic electrons to the interaction between the crystal and the beam of real photons with the angle divergence $\Delta \theta$ and the 'white' spectrum (1). In particular, the photons with the wavevector, which satisfies the Bragg condition for the crystal reciprocal-lattice vectors $\mathbf{H}$

$$
(\mathbf{k}+\mathbf{H})^{2} \simeq k^{2}, \quad 2 \omega(\mathbf{v H})+H^{2} c^{2} \simeq 0,
$$

will be reflected by the corresponding crystallographic planes and yields the peak of X-rays with the angle $2 \theta_{H}$ to the electron velocity. The value $\theta_{H}$ is the angle between the reflecting planes and the vector $\mathbf{v}$ (Fig. 1), that is,

$$
(\mathbf{v H})=-v H \sin \theta_{H} \text {. }
$$

The set of reflections distributed in space conditioned by various crystallographic planes represents the integral PXR spectrum from the ultrarelativistic electrons considered in detail by Feranchuk \& Ivashin (1985). The total intensity of the radiation emitted in every reflection is defined by the same structure amplitude $F(\mathbf{H})$ as the one defining the diffraction of real photons in the same direction. Moreover, every PXR reflection has the specific fine angular and frequency distributions near the angle $2 \theta_{H}$ and frequency $\omega_{H}$ (or wavelength $\lambda_{H}$ ) which can be calculated from (2):

$$
\omega_{H}=\frac{H c^{2}}{2 v \sin \theta_{H}} ; \quad \lambda_{H}=\frac{4 \pi v \sin \theta_{H}}{H c} .
$$

The above-mentioned features of PXR have been confirmed by numerous experimental results that are in a good agreement with the theoretical simulation (for example, Brenzinger et al., 1997, and references therein).

As follows from formula (3), the radiation frequency in the PXR reflection can be tuned by simple rotation of the crystal with respect to the electron beam. Usually, this property is considered as the basis for development of X-ray sources of monochromatic radiation with smoothly varied frequency (Feranchuk \& Ulyanenkov, 1999; Kaplin et al., 2002). However, in the present paper, we analyze this PXR feature in order to justify the possibility of direct measurement of the phase of the structure amplitude $F(\mathbf{H})$. Let us set the incidence angle of the electron beam in such a way that the radiation frequency of the PXR reflection from the investigated plane coincides with the frequency $\omega_{K}$ corresponding to the $K$ edge of the absorption band of one of the elements in the considered crystal:

$$
\sin \theta_{K}^{H}=\frac{H c^{2}}{2 v \omega_{K}}=\frac{c}{4 \pi v} H \lambda_{K},
$$

where $\lambda_{K}$ is the wavelength of the $K \alpha$ line for this element.

An analogous approach has been discussed in the paper by Feranchuk \& Ivashin (1989) for the case of dynamical diffraction and hard X-rays corresponding to the characteristic lines of heavy atoms. However, these conditions are not fulfilled for crystals of organic compounds, which are of most interest in the current investigation (see also Baryshevsky et al., 1983). Therefore, we estimate the typical parameters of the interaction between X-rays and organic crystals, i.e. the susceptibility $\chi_{0}$, the extinction length $L_{e}$ and the absorption 
length $L_{\text {abs }}$. For numerical calculations, the organic compound $\mathrm{C}_{13} \mathrm{H}_{14} \mathrm{~N}_{2} \mathrm{OS}$ is chosen, for which the structure has been recently solved by Gurskaya et al. (2003). The crystal possesses a monoclinic symmetry described by space group $P 2_{1} / c$ and includes 4 molecules in the elementary unit cell. Parameters of the cell $(a, b, c)$, the volume $\Omega$ and the angle $\beta$ are:

$$
\begin{gathered}
a=10.202, b=7.203, c=17.618 \AA ; \\
\Omega=1270 \AA^{3} ; \quad \beta=101.09^{\circ} .
\end{gathered}
$$

In accordance with (3), most PXR reflections for this crystal correspond to large wavelengths $\lambda \leq 40 \AA$. The wavelength of the $K$ edge of the $\mathrm{S}$ atom belongs to this range and is considered as the basis for the realization of the ASM scheme. Another reason for the selection of sulfur is the presence of this element in most organic compounds, thus isomorphic replacement is not required. The $\mathrm{S}$ atom also has a large nucleus charge and, therefore, its coordinates are well defined in X-ray structure analysis (Porai-Koshitz, 1968).

The total number of electrons in the elementary unit cell of the considered crystal is $Z_{\text {tot }}=4 \times 138=552$. The X-ray polarizability $\chi_{0}\left(\lambda_{1}\right)$ (James, 1960) at wavelength $\lambda_{1}=$ $\lambda_{K} / 1.1 \simeq 4.66 \AA$ near $\lambda_{K} \simeq 5.019 \AA$ corresponding to the $K$ edge of the absorption band of the $\mathrm{S}$ atom is

$$
\chi_{0}=-\frac{e^{2} Z_{\mathrm{tot}} \lambda_{1}^{2}}{4 \pi^{2} m c^{2} \Omega}
$$

and extinction and absorption lengths are equal:

$$
\begin{gathered}
\chi_{0} \simeq-7.98 \times 10^{-5}+i 3.47 \times 10^{-6} ; \\
L_{e}=\frac{\lambda_{1}}{\pi\left|\chi_{0}\right|} \simeq 1.86 \mu \mathrm{m} ; \quad L_{\mathrm{abs}} \simeq 21.4 \mu \mathrm{m} .
\end{gathered}
$$

The average effective charge for a single scattering atom in organic crystals is small enough (for the considered case $Z_{\text {eff }} \simeq 4$ ), therefore, electron multiple scattering in organic matter is essentially less than in inorganic crystals. The organic crystals are usually grown as thin films or mosaic crystals consisting of small blocks of thickness $L \sim L_{e}$ (Vainshtein, 1978). Thus, the angular and frequency distributions of PXR intensity emitted by one electron in the reflection with the vector $\omega_{H} \mathbf{v} / c^{2}+\mathbf{H}$ can be calculated in the framework of the kinematic approximation. The frequency of $\mathrm{X}$-rays in the reflection unambiguously depends on the exit angle of the photons because of the condition (2), and the angular distribution within the reflection is defined by the following formula (Feranchuk \& Ivashin, 1985):

$$
\begin{aligned}
\frac{\partial^{2} N_{n}}{\partial \theta_{x} \partial \theta_{y}}= & \frac{e^{2}}{4 \pi \hbar c^{2}} \frac{\omega_{\theta}^{(n)} L_{\text {abs }}}{\sin ^{2} \theta_{H}}\left[1-\exp \left(-\frac{L}{L_{a b s}}\right)\right] \frac{\left[\theta_{x}^{2} \cos ^{2} 2 \theta_{H}+\theta_{y}^{2}\right]}{\left[\theta_{x}^{2}+\theta_{y}^{2}+\theta_{p h}^{2}\right]^{2}} \\
& \times\left|\chi_{\mathbf{H}}\left(\omega_{\theta}^{(n)}\right)\right|^{2} .
\end{aligned}
$$

These PXR photons are related to the following set of reciprocal-lattice vectors:

$$
\mathbf{H}_{n}=\frac{2 \pi n}{d} \mathbf{Z}, \quad n=1,2, \ldots,
$$

where $d$ is the minimal distance between the crystallographic planes along the normal unit vector $\mathbf{Z}$. The angles $\theta_{x}, \theta_{y}$ are referred to the plane perpendicular to the vector $\omega_{H} \mathbf{v} / c^{2}+\mathbf{H}$ (Fig. 1). The values $\theta_{x}, \theta_{y}$ are small because the angular width of the reflection is defined by the parameter (the electron multiple scattering is neglected):

$$
\theta_{\mathrm{ph}}=\left(\frac{m c^{2}}{E}+\left|\chi_{0}\right|\right)^{1 / 2} \simeq 10^{-2} .
$$

The fine angular distribution within the PXR reflection, being calculated by means of formula (8), are observed by several experimental groups, for example, Brenzinger et al. (1997). The frequency spectrum of the radiation concentrated in the considered direction is represented by the set of sharp spectral lines (harmonics) corresponding to the following frequencies:

$$
\omega_{H}^{(n)}=\frac{\pi n c^{2}}{v d \sin \theta_{H}} .
$$

The intensity of the harmonics decreases with the harmonics number as

$$
\frac{I_{n}}{I_{1}} \simeq \frac{\omega_{H}^{(n)}\left|\chi_{\mathbf{H}}\left(\omega_{H}^{(n)}\right)\right|^{2}}{\omega_{H}^{(1)}\left|\chi_{\mathbf{H}}\left(\omega_{\theta}^{(1)}\right)\right|^{2}} \simeq \frac{1}{n^{3}} .
$$

Therefore, the main contribution to the reflection intensity is defined by the first harmonics, if it is not forbidden. The spectral width can be calculated from Bragg condition (2), taking into account a deviation of the wavevector of the emitted photon from the direction $\omega_{H} \mathbf{v} / c^{2}+\mathbf{H}$. The unit vectors $\mathbf{e}_{x}, \mathbf{e}_{y}$, being introduced in the plane of the detector in such a way that $\mathbf{e}_{x}$ is in the plane of the vectors $\mathbf{v}, \mathbf{H}$ and $\mathbf{e}_{y}$ is perpendicular to this plane (Fig. 1), transform equation (2) into the following form:

$$
2 \omega_{\theta}^{(n)}\left[\mathbf{v H}+c\left(\mathbf{e}_{x} \theta_{x}+\mathbf{e}_{y} \theta_{y}\right) \mathbf{H}\right]+H^{2} c^{2}=0 .
$$

The approximate solution for this equation in the case of small angles $\theta_{x}, \theta_{y}$ delivers a fine structure of the frequency spectrum for $n$th harmonics:

$$
\omega_{\theta}^{(n)} \simeq \omega_{H}^{(n)}\left(1-\frac{c}{v} \theta_{x} \cot \theta_{H}\right) .
$$

Thus, the relative spectral width of the harmonics is defined by the same parameter $\theta_{\mathrm{ph}}$ as its angular width:

$$
\left(\frac{\Delta \omega}{\omega}\right)_{\mathrm{PXR}} \simeq \theta_{\mathrm{ph}} .
$$

To perform an integration over the angles in formula (8), the dependence of susceptibility on frequency and angle due to equation (13) should be taken into account. This dependence can be in the obvious form (Authier, 2001):

$$
\begin{aligned}
\chi_{\mathbf{H}}(\omega)= & -\frac{4 \pi r_{e}}{\omega^{2} \Omega} F(\mathbf{H}) \\
F(\mathbf{H}, \omega)= & \sum_{j}\left[f_{0 j}(\mathbf{H})+f_{j}^{\prime}(\omega)+i f_{j}^{\prime \prime}(\omega)\right] \exp \left[-W_{j}(\mathbf{H})\right] \\
& \times \exp \left(i \mathbf{H} \cdot \mathbf{R}_{j}\right) .
\end{aligned}
$$

Here $r_{e}$ is the classical radius of the electron, $\Omega$ is the volume of the crystallographic unit cell, $f_{0 j}(\mathbf{H})$ is the atomic scattering factor of the $j$ th atom corresponding to the wavevector transfer $\mathbf{H}, \exp [-W(\mathbf{H})]$ is the Debye-Waller factor, $f_{j}^{\prime}(\omega)$, 
$f_{j}^{\prime \prime}(\omega)$ are the anomalous dispersion and absorption corrections. The structure phase factor $\exp \left(i \mathbf{H} \mathbf{R}_{j}\right)$ is defined by the coordinate $\mathbf{R}_{j}$ of the atom in the unit cell, $F(\mathbf{H})$ is the total structure amplitude normalized as $F(0)=Z_{\text {tot }}$. In the range of the PXR harmonic spectral width, the essential dependence on the frequency in the formula (15) is defined by the functions $f^{\prime}, f^{\prime \prime}$. This dependence is quite universal if the anomalous corrections are normalized on the value $f_{0}$ and should be taken into account near the frequency $\omega \simeq \omega_{K}$ of the absorption edge (Vainshtein, 1978). Fig. 2 shows the relative width of the absorption edge, which is essentially more than the characteristic width of the PXR reflection $\Delta \omega / \omega_{K} \simeq$ $1 \gg \theta_{\mathrm{ph}}$. Therefore, the susceptibility $\chi_{\mathbf{H}}(\omega)$ can be considered as a constant value with the argument $\omega=\omega_{H}^{(n)}$ when calculating the integral in formula (8). The value $f^{\prime \prime}(\omega)$ is not well defined at the angle $\theta_{H}$, which corresponds to the condition $\omega_{H}^{(n)}=\omega_{K}$. However, the applications considered in this paper correspond to the frequency range

$$
1.1<\omega_{H}^{(n)} / \omega_{K}<1.5,
$$

where the anomalous corrections give an important contribution to the structure amplitudes, changing smoothly within the limits of the PXR reflections.

After integration over the angles in the formula (8), the intensity of the photons $I_{n}$ emitted in single PXR harmonics can be calculated for the electron current $J$ passing through the investigated crystal (Feranchuk \& Ivashin, 1985) as

$$
\begin{aligned}
I_{n}= & A\left(E, \omega_{H}^{(n)}\right)\left|F\left(\mathbf{H}_{n}, \omega_{H}^{(n)}\right)\right|^{2} ; \\
A\left(E, \omega_{H}^{(n)}\right)= & \frac{e^{2} 2 \pi^{2} r_{e}^{2}}{\left(\omega_{H}^{(n)}\right)^{3} \Omega^{2} \hbar c^{4}} \frac{\left(1+\cos ^{2} \theta_{H}\right) L_{\mathrm{abs}}}{\sin ^{2} \theta_{H}} \\
& \times\left[1-\exp \left(-\frac{L}{L_{\mathrm{abs}}}\right)\right] \\
& \times\left[\ln \left(\frac{\theta_{D}^{2}+\theta_{\mathrm{ph}}^{2}}{\theta_{\mathrm{ph}}^{2}}\right)-\frac{\theta_{D}^{2}}{\theta_{D}^{2}+\theta_{\mathrm{ph}}^{2}}\right] \frac{J}{e} .
\end{aligned}
$$

Here the parameter $\theta_{D} \sim \theta_{\mathrm{ph}}$ defines the angular width of the detector and the harmonics spectral width in accordance with formula (13). The spectral resolution of the detector is

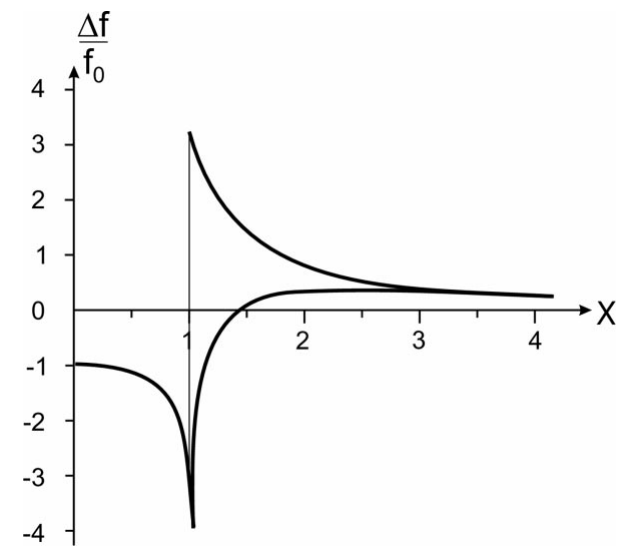

Figure 2

Imaginary (upper curve) and real (lower curve) anomalous corrections in the range of the absorption edge supposed to be sufficient to measure separately every PXR harmonic. The value $A\left(E, \omega_{H}^{(n)}\right)$ in formula (17) describes the kinematics of the radiation process and does not depend on the structure amplitude $F$.

Using fundamental constants, formula (17) can be represented in the form that permits the rate of the photon counts to be estimated in real experiments:

$$
\begin{aligned}
I_{n}\left[\text { photons s }^{-1}\right] \simeq & 5.6 \times 10^{7} \frac{1}{\left(\omega_{H}^{(n)}\right)^{3} \Omega^{2}} K\left|F\left(\mathbf{H}_{n}, \omega_{H}^{(n)}\right)\right|^{2} J[\mu \mathrm{A}] ; \\
K= & \frac{\left(1+\cos ^{2} \theta_{H}\right) L_{\mathrm{abs}}}{\sin ^{2} \theta_{H}}\left[1-\exp \left(-\frac{L}{L_{\mathrm{abs}}}\right)\right] \\
& \times\left[\ln \left(\frac{\theta_{D}^{2}+\theta_{\mathrm{ph}}^{2}}{\theta_{\mathrm{ph}}^{2}}\right)-\frac{\theta_{D}^{2}}{\theta_{D}^{2}+\theta_{\mathrm{ph}}^{2}}\right] .
\end{aligned}
$$

The radiation frequency is measured in $\mathrm{keV}$, the volume of the crystallographic unit cell is defined in $\AA^{3}$, the absorption length and the crystal thickness are measured in $\mu \mathrm{m}$, and $K$ is approximately equal to unity. For the crystal $\mathrm{C}_{13} \mathrm{H}_{14} \mathrm{~N}_{2} \mathrm{OS}$ of thickness $L=10 \mu \mathrm{m}$ and X-radiation close to the $K \alpha$ line of the $\mathrm{S}$ atom for reflection with $F(\mathbf{H}) \sim 20$, formula (17) gives an estimate

$$
I_{n}\left[\text { photons s}^{-1}\right] \simeq 10^{4} J[\mu \mathrm{A}] .
$$

The success of experimental realization of the considered method depends on the ratio of the X-ray intensity in the PXR reflection (signal) to the intensity of X-rays emitted by the electrons due to other processes that are not strictly connected to the structure amplitudes (noise). The Bremsstrahlung is the process contributing the radiation background to the continuous spectrum due to the interaction between electrons and the medium. In the case of ultrarelativistic electrons, the total flux of the Bremsstrahlung is concentrated in a narrow cone with divergence angle $\sim \theta_{\mathrm{ph}}$ along the particle velocity. Therefore, the photons emitted in PXR reflections at large angles with respect to the electron velocity are registered with low background, as confirmed in several experiments (Brenzinger et al., 1997).

The only competing process for PXR in the range of the anomalous scattering is characteristic radiation $(\mathrm{ChR})$, which is caused by excitation of the crystal atoms by the electron beam. This radiation is represented by a set of narrow spectral lines and has almost isotropic angular distribution (Krause \& Oliver, 1979). In most cases, the relative spectral width of the ChR lines is essentially less than the width of PXR harmonics defined by formula (14):

$$
(\Delta \omega / \omega)_{\mathrm{ChR}} \ll(\Delta \omega / \omega)_{\mathrm{PXR}} .
$$

If the photons are detected by an X-ray spectrometer with energy resolution of the same order as the spectral width of the PXR reflection (14), the intensities of PXR and ChR can be independently measured in the frequency range (16). To compare the radiation intensities for both mechanisms, the experimental data for the photo-ionization $\sigma_{\text {ph }}$ (Yeh \& Lindau, 1985) and for the fluorescence yield $Y_{f}(Z)$ (Krause, 1979) are used for the calculation of probability of an exci- 
tation of atom $\mathrm{S}$ by the electron beam $(Z$ is the atom nucleus charge). The expression (1) is used for calculation of spectral density of pseudo-photons corresponding to one electron. Then the intensity of ChR quanta generated from the crystal with thickness $L$ and density of the $\mathrm{S}$ atoms $n_{\mathrm{S}}$ by the electron current $J$ and registered by the detector with the angular resolution $\theta_{D}$ can be calculated as follows:

$$
I_{\mathrm{ChR}} \simeq \int_{\omega_{\min }}^{\infty} \mathrm{d} \omega n(\omega) \sigma_{\mathrm{ph}}(\omega) Y_{f}(Z) n_{\mathrm{S}} L \frac{\theta_{D}^{2}}{2} \frac{J}{e} .
$$

Experimental data for the value $\sigma_{\mathrm{ph}}$ of the $\mathrm{S}$ atom (Yeh \& Lindau, 1985) can be interpolated by the analytical formula

$\sigma_{\mathrm{ph}}(\omega) \simeq \sigma_{0}\left(\frac{\omega_{\min }}{\omega}\right)^{2}, \quad \sigma_{0} \simeq 0.7 \times 10^{-22} \mathrm{~m}^{2}, \quad h \omega_{\min } \simeq 150 \mathrm{eV}$

and the value $Y_{f}(16) \simeq 0.1$ (Krause, 1979). We choose the same values for other physical parameters as in expression (19):

$$
n_{S}=\frac{1}{\Omega}, \quad \Omega=1270 \AA^{3}, \quad L=10 \mu \mathrm{m}, \quad \theta_{D}=10^{-2} .
$$

Finally,

$$
I_{\mathrm{ChR}}\left[\text { photons s}^{-1}\right] \simeq 2.1 \times 10^{4} J[\mu \mathrm{A}] .
$$

Thus, the intensities of ChR and PXR have the same order of magnitude for the considered experimental conditions. The high intensity of PXR is essential for the following experimental aspect of the problem. The anomalous scattering corrections for the $\mathrm{S}$ atom have the values $3<\left|\Delta f^{\prime}\right| \sim \Delta f^{\prime \prime}<5$ (Mirkin, 1961) for the frequency range (16), which corresponds to several percent of the full structure amplitude of typical organic crystals. Therefore, the use of the ASM requires accurate measurement of the intensity of PXR reflections, which is also the case for standard X-ray structure analysis (Porai-Koshitz, 1968). Accuracy of the intensity measurements is mainly defined by the fluctuations of the counting rate, which produces a restriction for minimal observation time $t_{\min }$. In particular, in order to measure the

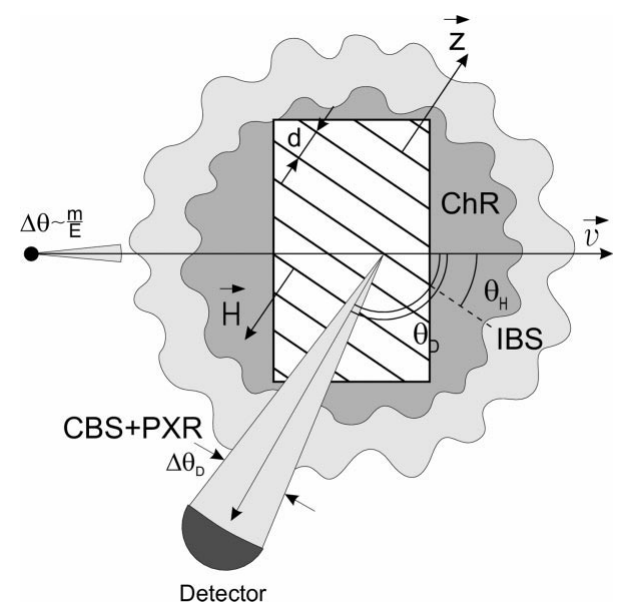

Figure 3

Characteristic angular distributions of various X-radiations in the case of nonrelativistic electrons structure amplitude with the relative accuracy $\delta_{F}$, the value $t_{\min }$ should be of order (Feranchuk \& Ivashin, 1989)

$$
t_{\min } \simeq 1 / I_{n} \delta_{F}^{2}
$$

where $I_{n}$ is the X-ray intensity.

\section{Anomalous scattering method using the PXR from non-relativistic electrons}

Coherent X-rays emitted in crystals by electrons of low energy ( $\sim 50-100 \mathrm{keV})$ have several qualitative features, which are absent in PXR from ultrarelativistic electrons. Emission of this radiation has been recently considered in detail in our papers (Feranchuk \& Ulyanenkov, 1999; 2001a,b; Feranchuk et al., 2000) and here only the essential features are given.

Firstly, in the non-relativistic case, no kinematic factor exists that selects the direction along the particle velocity for the radiation angular distribution. Therefore, the X-rays produced by electrons via all essential mechanisms [PXR, coherent Bremsstrahlung (CB), incoherent Bremsstrahlung (IBS) and $\mathrm{ChR}$ ] are distributed almost isotropically (Fig. 3). PXR and $\mathrm{CB}$ both depend on the structure amplitudes of the elementary unit cell due to the coherent summation of the radiation amplitudes from the periodically ordered atoms of the crystal. Besides, the amplitudes of the two processes interfere and cannot be considered independently, and their resulting intensity represents a new radiation mechanism that is called coherent X-radiation (CXR) (Blazhevich et al., 1994; Morokhovsky et al., 2000; Feranchuk et al., 2000). The spectralangular distribution of the photons with polarization $\mathbf{e}_{s}$ and wavevector $\mathbf{k}=\omega \mathbf{n} / c$ radiated into the $n$th CXR harmonics is defined by the following formula (Feranchuk et al., 2000; Feranchuk \& Ulyanenkov, 2001a):

$$
\begin{aligned}
\frac{\partial^{2} N_{n s}}{\partial \mathbf{n} \partial \omega}= & \frac{e^{2}}{2 \pi \hbar c^{3}} \frac{\omega_{\theta}^{(n)} L_{\mathrm{abs}}}{v}\left[1-\exp \left(-\frac{L}{L_{\mathrm{abs}}}\right)\right] \\
& \times\left|A_{\mathrm{PXR}}+A_{\mathrm{CB}}\right|^{2} \delta[\omega-\mathbf{v}(\mathbf{k}+\mathbf{H})], \\
A_{\mathrm{PXR}}= & \frac{\chi_{\mathbf{H}}}{k_{H}^{2}-\omega^{2} / c^{2}}\left[\left(\mathbf{v k}_{H}\right)\left(\mathbf{e}_{s} \mathbf{H}\right)-\omega^{2}\left(\mathbf{e}_{s} \mathbf{v}\right) / c^{2}\right], \\
A_{\mathrm{CB}}= & -\frac{e U_{\mathbf{H}}}{m \Omega\left(\mathbf{v k}_{H}\right)}\left[\left(\mathbf{e}_{s} \mathbf{H}\right)+\frac{\left(\mathbf{e}_{s} \mathbf{v}\right)(\mathbf{k H})}{(\mathbf{v k})}\right], \\
\mathbf{k}_{H}= & \mathbf{k}+\mathbf{H}, \quad \mathbf{H} \rightarrow \mathbf{H}_{n}=\frac{2 \pi n}{d} \mathbf{Z},
\end{aligned}
$$

which is in fact a non-relativistic analogue of formula (8). The total CXR intensity contains two terms with different physical meanings. $A_{\mathrm{PXR}}$ is the amplitude of the parametric X-radiation, which is the diffraction of the electron's own electromagnetic field in the crystal, analogously to the ultrarelativistic case. This contribution [see equation (15)] depends on the crystal susceptibility $\chi_{\mathbf{H}}$, which includes the structure amplitude $F(\mathbf{H})$ and is defined by the electron density in the unit cell. Coherent Bremsstrahlung is produced by the diffraction of the electrons due to their interaction with both electrons and nuclei of the crystal. Therefore, the amplitude $A_{\mathrm{CB}}$ includes the Fourier component of the full atomic potential: 


$$
\begin{aligned}
U_{\mathbf{H}} & =\frac{4 \pi e}{H^{2}}\left[F_{0}(\mathbf{H})-F(\mathbf{H})\right] \\
F_{0}(\mathbf{H}) & =\sum_{j} Z_{j} \exp \left[-W_{j}(\mathbf{H})\right] \exp \left(i \mathbf{H} \mathbf{R}_{j}\right) .
\end{aligned}
$$

Thus, in the non-relativistic case the CXR intensity includes the additional structure amplitude $F_{0}(\mathbf{H})$, which depends only on the coordinates of the nuclei in the elementary unit cell (Feranchuk \& Ulyanenkov, 2001a).

The CXR photons can be radiated in any direction, and the argument of the $\delta$ function in formula (24) defines the condition of coherent radiation. Unlike the ultrarelativistic case (10), the frequency of CXR photons depends not only on the angle between the electron velocity and crystallographic plane but also on the angle $\theta$ between the velocity and direction to detector (Fig. 3):

$$
\omega_{H}^{(n)}(\theta)=\frac{2 \pi v \cos \theta_{H}}{d(1-v / c \cos \theta)} n .
$$

When the observation angle $\theta$ is fixed, the CXR spectral width is defined by the length of the coherent interaction between the electron and crystal [before the multiple scattering becomes essential (Feranchuk \& Ivashin, 1985)]. The multiple scattering can be large for non-relativistic electrons, however, the averaged nucleus charge is small in organic crystals, and estimation of the multiple scattering length $L_{\mathrm{sc}}$ gives (TerMikaelyan, 1972)

$$
L_{\mathrm{sc}} \sim 1-10 \mu \mathrm{m} .
$$

Thus, the effective application of the ASM with non-relativistic electrons is possible for monocrystal films with thickness $L<L_{\mathrm{sc}}$. In this case, the relative spectral width of the CXR harmonics is defined by the coherent interaction of the electrons with the crystal and follows from the expression

$$
\frac{\Delta \omega_{0}}{\omega} \simeq \frac{v}{L \omega_{H}^{(n)}(\theta)} .
$$

The photons emitted in the fixed direction have a high monochromaticity. For example, for the sulfur sample with $L=10 \mu \mathrm{m}$ and $\omega_{H}^{(n)}=\omega_{K}$,

$$
\Delta \omega_{0} / \omega \simeq 10^{-4} .
$$

However, in real experiments, the observed CXR line width is defined by angular $\Delta \theta$ and frequency $\Delta \omega \gg \Delta \omega_{0}$ resolutions of the photon detector. According to Feranchuk \& Ulyanenkov (2001a), this dependence can be taken into account as a normalized apparatus function substituting for the $\delta$ function in the formula (24):

$$
\begin{aligned}
\Phi & {\left[\omega-\omega_{H}^{(n)}\left(\theta_{0}\right)\right] } \\
& =\Delta \theta^{2} \exp \left[-\frac{\left(\omega-\omega_{H}^{(n)}\right)^{2}}{\Delta \omega^{2}}-\frac{\left(\omega_{H}^{(n)} v \Delta \theta\right)^{2}}{4 \Delta \omega^{2} c^{2}\left(1-v / c \cos \theta_{0}\right)}\right],
\end{aligned}
$$

where angle $\theta_{0}$ defines the direction to the center of the detector (Fig. 3).

We suppose here that angular and spectral resolutions of the X-ray detector are sufficient to select single CXR harmonics. Then, summing up the polarizations and inte- grating over the angle and frequency in formula (24), the number of quanta registered by the detector in the CXR reflection and for electron current $J$ can be found:

$$
\begin{aligned}
I_{n}= & B\left(v, H_{n}\right)\left\{\left|F\left(\mathbf{H}_{n}, \omega^{(n)}\right)\right|^{2} \frac{q^{4}}{\left[1+\beta \cos \left(\theta_{H}+\theta_{0}\right)\right]^{2}}\right. \\
& \times\left[\sin \left(\theta_{H}+\theta_{0}\right)-\frac{\beta^{2} \cos \theta_{H} \sin \theta_{0}}{q}\right]^{2} \\
& +\left|F\left(\mathbf{H}_{n}, \omega^{(n)}\right)+F_{0}\left(\mathbf{H}_{n}, \omega^{(n)}\right)\right|^{2} \\
& \times\left[\sin \left(\theta_{H}+\theta_{0}\right)-\frac{\beta \cos \left(\theta_{H}+\theta_{0}\right) \sin \theta_{0}}{q}\right]^{2} \\
& -2 \Re\left\{F^{*}\left(\mathbf{H}_{n}, \omega^{(n)}\right)\left[F\left(\mathbf{H}_{n}, \omega^{(n)}\right)-F_{0}\left(\mathbf{H}_{n}, \omega^{(n)}\right)\right]\right\} \\
& \times\left[\sin ^{2}\left(\theta_{H}+\theta_{0}\right)\right. \\
& +\frac{\beta \sin \left(\theta_{H}+\theta_{0}\right) \sin \theta_{0}\left[\cos \left(\theta_{H}+\theta_{0}\right)-\beta \cos \theta_{H}\right]}{q} \\
& \left.\left.-\frac{\beta^{3} \cos \theta_{H} \sin ^{2} \theta_{0} \cos \left(\theta_{H}+\theta_{0}\right)}{q^{2}}\right]\right\}, \\
\beta= & v / c, \quad q=1-\beta \cos \theta_{0}, \\
B\left(v, H_{n}\right)= & \frac{e^{2} 8 \pi^{2} r_{e}^{2} L}{\beta^{2} \cos \theta_{H} q H_{n}^{3} \Omega^{2} \hbar c} \Delta \theta^{2} \frac{J}{e} .
\end{aligned}
$$

This expression is equivalent to formula (17) for the nonrelativistic case, and can be used for a crystal thickness less than the absorption length $\left(L \ll L_{\text {abs }}\right)$ and angular resolution of the detector less than its relative spectral resolution $(\Delta \theta<\Delta \omega / \omega)$. In order to analyze the CXR spectra in the anomalous scattering range, the angles $\theta_{H}, \theta_{0}$ have to be related, as follows from equation (26):

$$
\omega_{K} d(1-\beta \cos \theta) \simeq 2 \pi v n \cos \theta_{H} .
$$

As shown recently for non-relativistic electrons (Feranchuk \& Ulyanenkov, 2001a), both structure amplitudes $\left|F\left(\mathbf{H}_{n}\right)\right|$, $\left|F_{0}\left(\mathbf{H}_{n}\right)\right|$ and their relative phase can be measured separately even out of the anomalous scattering range if crystal orientation or detector angle are varied. The intensity of photons follows from formula (29) with the same crystal parameters as in formula (19) and the electron energy $E=100 \mathrm{keV}$ :

$$
I_{n}\left[\text { photons }^{-1}\right] \simeq 10^{2} J[\mu \mathrm{A}] .
$$

This value is essentially less than in the case of ultrarelativistic particles. However, such radiation has a great advantage for applications because it does not require bright synchrotron sources and can be used in a laboratory using a source with a high electron current.

Another factor defining the accuracy of the experimental measurement of structure amplitudes is the ratio of signal (CXR intensity) to noise (the characteristic radiation and Bremsstrahlung). The intensity of the characteristic radiation does not depend essentially on the electron energy, and follows from equation (22), which results for nonrelativistic particles in

$$
I_{\mathrm{ChR}} \gg\left(I_{n}\right)_{\mathrm{CXR}}
$$


Thus, the ASM on the basis of CXR requires an X-ray detector with rather good spectral resolution of $\Delta \omega / \omega_{K} \simeq 0.1$, which should allow measurement of CXR and ChR lines separately. Bremsstrahlung in the direction of the CXR reflection is an additional source of noise in the case of nonrelativistic electrons (Fig. 3). In a previous paper (Feranchuk et al., 2000), the ratio of the CXR intensity to the BS intensity was estimated as

$$
\xi=\frac{\left(I_{n}\right)_{\mathrm{CXR}}}{I_{\mathrm{BS}}} \simeq \frac{6 \pi^{2} v}{\Omega \omega_{K}^{3} \ln \left[137 / Z_{\mathrm{eff}}^{1 / 3}\right]} \frac{\omega_{K}}{\Delta \omega},
$$

and it also depends on the detector spectral resolution. Here $Z_{\text {eff }}$ is the effective charge for one atom in the crystal unit cell. In particular, for the organic crystal $\mathrm{C}_{13} \mathrm{H}_{14} \mathrm{~N}_{2} \mathrm{OS}, \xi \simeq 10$ for detector resolution $\Delta \omega / \omega_{K} \simeq 0.1$.

\section{Phase simulation}

As follows from the preceding sections, the intensity of X-ray reflections resulting from the coherent interaction of the electron beam with an investigated crystal can be accurately measured in the vicinity of the absorption edge of one of the atoms of a crystallographic unit cell. This fact can be used for direct measurement of the phase of structure amplitudes. Up to now, the experimental investigations of the PXR spectra concern mainly the optimization of conditions for use of PXR as the source of the quasi-monochromatic X-rays. Therefore, the existing experimental data cannot be used for illustration of the theoretical analysis considered above, and we provide a simulation in order to find the experimental conditions for realization of the ASM on the basis of PXR.

The structure of the organic crystal $\mathrm{C}_{13} \mathrm{H}_{14} \mathrm{~N}_{2} \mathrm{OS}$ has already been solved (Gurskaya et al., 2003), and the real values of the structure amplitude modulus $\left|F_{0}(\mathbf{H})\right|$ and its phase $\varphi_{0}(\mathbf{H})$ can therefore be calculated for reciprocal-lattice vector $\mathbf{H}=(h, k, l)=(1,1,3)$. When this amplitude is calculated (James, 1960) for the wavelength $\lambda$ essentially different from the $K$ edge $\lambda_{K}$ of the absorption edge of any atom in the crystal unit cell, the anomalous scattering corrections are small and the structure amplitudes do not depend on the wavelength but are defined by the electron-density distribution only. The following values are calculated for the above-mentioned reflection and $\lambda=1.54 \AA$ (Feranchuk et al., 2002):

$$
\begin{gathered}
F_{0}(1,1,3)=F_{0}^{\prime}+i F_{0}^{\prime \prime}=11.79-i 0.170, \\
\left|F_{0}\right|=11.79, \quad \varphi_{0}=\arctan \frac{F_{0}^{\prime \prime}}{F_{0}^{\prime}}=-0.014 .
\end{gathered}
$$

When the ASM is used, the modulus of the structure amplitude $\left|F_{0}(\mathbf{H})\right|$ is supposed to be known (Woolfson \& Fan, 1995) because it can be measured from either the corresponding diffraction intensity or from the PXR intensity in the same reflection but far from the absorption edge. The structure factor $|S(\mathbf{H})|$ and its phase $\psi(\mathbf{H})$ for the $\mathbf{S}$ atom in the crystallographic unit cell are defined in the following way:

$$
F_{\mathrm{S}}(\mathbf{H})=f_{\mathrm{S}}(\mathbf{H}) S(\mathbf{H}), \quad S(\mathbf{H})=\exp \left[-W_{\mathrm{S}}(\mathbf{H})\right] \sum_{s} \exp \left(i \mathbf{H} \mathbf{R}_{s}\right)
$$

where $f_{\mathrm{s}}(\mathbf{H})$ and $\exp \left[-W_{\mathrm{S}}(\mathbf{H})\right]$ are the atomic scattering factor and Debye-Waller factor of the S atom, respectively, and the structure factor $S(\mathbf{H})$ is defined by the coordinates $\mathbf{R}_{s}$ of these atoms in the elementary cell. For the considered reflection $\mathbf{H}=(1,1,3)$ and wavelength $1.54 \AA$, these values are equal to

$$
\begin{gathered}
f_{\mathrm{S}}(\mathbf{H})=1.718-i 0.069, \quad \exp \left[-W_{S} \mathrm{~S}(\mathbf{H})\right]=0.94, \\
|S(\mathbf{H})|=0.133, \quad \psi(\mathbf{H}) \simeq 0 .
\end{gathered}
$$

Usually the modulus and phase of the structure factor $F_{\mathrm{S}}(\mathbf{H})$ can be considered as known because the positions of most heavy atoms in the unit cell are defined accurately during the primary analysis of the Patterson function (Porai-Koshitz, 1968). This assumption on known values $|S(\mathbf{H})|, \psi(\mathbf{H})$ actually means that unknown phases are calculated relative to the phases corresponding to the distribution of the anomalously scattering atoms in the unit cell (Vainshtein, 1978). Therefore, the phase $\psi(\mathbf{H})$ can be substituted as the zero value.

Now the structure amplitudes may be calculated taking into account the anomalous scattering corrections for the $\mathrm{S}$ atom and the X-ray frequencies $\omega_{1}=1.1 \omega_{K}, \omega_{2}=1.5 \omega_{K}$. The results are the following:

$$
\begin{aligned}
F\left(\mathbf{H}, \omega_{1,2}\right) & =\left|F_{0}(\mathbf{H})\right|\left(\cos \varphi_{0}+i \sin \varphi_{0}\right)+\Delta F_{1,2}^{\prime}+i \Delta F_{1,2}^{\prime \prime}, \\
\Delta F_{1,2} & =\Delta f_{\mathrm{s}}\left(\omega_{1,2}\right) S(\mathbf{H}), \\
\Delta F_{1}^{\prime} & =-0.696, \quad \Delta F_{2}^{\prime}=0.279, \\
\Delta F_{1}^{\prime \prime} & =-1.202, \quad \Delta F_{2}^{\prime \prime}=-0.622 .
\end{aligned}
$$

In order to extract the anomalous-scattering corrections for the considered reflection, the structure-amplitude modulus should be measured with an accuracy better than $\delta \simeq 10 \%$.

Let us now simulate the PXR characteristics for this reflection, considering the radiation from the electron beam with the energy $E=30 \mathrm{MeV}$ and current $J=1 \mu \mathrm{A}$. The observation angles $\theta_{1,2}$, which allow the intensity of the radiation to be measured with two different frequencies are calculated by means of the formula (4):

$$
\theta_{1,2}=\arcsin \left(\frac{H c^{2}}{2 v \omega_{1,2}}\right), \quad \theta_{1}=34.6^{\circ}, \quad \theta_{2}=24.6^{\circ} .
$$

These peaks are easily separated with necessary accuracy.

In some cases, it can be difficult to measure PXR intensities for two positions of the detector close to each other in the limits of the angular width of one reflection corresponding to the definite reciprocal-lattice vector $\mathbf{H}$. In this situation, the measurements for the fixed frequency except for two different reflections corresponding to the reciprocal-lattice vectors $\mathbf{H}$ and $-\mathbf{H}$ can be performed. This approach was usually considered for the ASM in the conventional diffraction experiments (Porai-Koshitz, 1968), and it is possible for a crystal with a unit cell without a center of symmetry $\left(\varphi_{0} \neq 0, \pi\right)$. In this case, the geometry of the PXR experiment should be inverted relative to the electron velocity in comparison with Fig. 1 and the amplitude $F\left(-\mathbf{H}, \omega_{1}\right)$ is calculated by means of the formula 


$$
F\left(-\mathbf{H}, \omega_{1}\right)=\left|F_{0}(\mathbf{H})\right|\left(\cos \varphi_{0}-i \sin \varphi_{0}\right)+\Delta F_{1}^{\prime}+i \Delta F_{1}^{\prime \prime} .
$$

Now we can use the values of the structure amplitudes (34)(38) in formula (17) for calculation of the PXR intensities. In accordance with (36), for reflection 113 , the phase $\varphi_{0}$ is very close to zero because of the symmetry of the considered structure and therefore the use of the reflection $\overline{1} \overline{1} \overline{3}$ is not effective. However, PXR intensities for two close frequencies [observation angles according to (38)] for the same reflection can be measured and the calculation results in the following values:

$$
\begin{aligned}
& \left.I_{\mathbf{H}, \omega_{1}}=1.28 \times 10^{4} \text { photons s }^{-1}\right], \\
& \left.I_{\mathbf{H}, \omega_{2}}=1.08 \times 10^{4} \text { photons s }^{-1}\right] .
\end{aligned}
$$

Here the value $L=L_{\text {abs }}=10 \mu \mathrm{m}$ for the crystal thickness is used; $\theta_{D}=10^{-2}$ and the same values for the current and energy of the electron beam as in (37). The magnitude of the intensity is weakly sensitive to the accuracy of the definition of the experimental parameter $\theta_{D}$, since it is included in the argument of the logarithmic function only. If the considered crystal is also characterized by some mosaicity parameter $\delta$, this parameter should be included in formula (17) along with the value $\theta_{D}$ when calculating the PXR intensity (Feranchuk \& Ivashin, 1985). Thus, the PXR intensities should be measured with a relative accuracy of $15 \%$. According to (23), such accuracy can be obtained for observation time $t_{\mathrm{obs}} \simeq 20 \mathrm{~s}$ if the photon intensity is given by (40).

For investigations of structures with a center of symmetry, it is important to distinguish whether the structure amplitude has the phase $\varphi_{0}=0$ or $\varphi_{0}=\pi$ (Vainshtein, 1978). The considered approach could solve this problem as well. If the phase $\varphi_{0}$ in (37) is assumed to be equal to $\pi$ instead of zero, the correlation between PXR intensities changes essentially:

$$
\begin{aligned}
& \left.I_{\mathbf{H}, \omega_{1}}^{(-)}=1.02 \times 10^{4} \text { photons s }^{-1}\right], \\
& \left.I_{\mathbf{H}, \omega_{2}}^{(-)}=1.19 \times 10^{4} \text { photons s }^{-1}\right] .
\end{aligned}
$$

In a real experiment with the application of PXR for the ASM, the main goal is to evaluate the phase $\varphi_{0}$, which means that the inverse problem should be solved. The modulus of the structure amplitudes has to be calculated on the basis of the intensity measurements for two different positions of the detector:

$$
\left|F\left(\mathbf{H}, \omega_{1,2}\right)\right|=\left[I_{\mathbf{H}, \omega_{1,2}} A\left(E, \omega_{1,2}\right)\right]^{1 / 2},
$$

with the function $A(E, \omega)$ defined by formula (17). Then the unknown phase $\varphi_{0}$ can be calculated as the solution of the following equations, which follow from (37):

$$
\begin{aligned}
\sin \varphi_{0}(\mathbf{H}) & =\frac{Q_{1} \Delta F_{2}^{\prime}-Q_{2} \Delta F_{1}^{\prime}}{2\left|F_{0}(\mathbf{H})\right| P} \\
\cos \varphi_{0}(\mathbf{H}) & =-\frac{Q_{1} \Delta F_{2}^{\prime \prime}-Q_{2} \Delta F_{1}^{\prime \prime}}{2\left|F_{0}(\mathbf{H})\right| P}, \\
Q_{1,2} & =\left|F\left(\mathbf{H}, \omega_{1,2}\right)\right|^{2}-\left|F_{0}(\mathbf{H})\right|^{2}-\left(\Delta F_{1,2}^{\prime}\right)^{2}-\left(\Delta F_{1,2}^{\prime \prime}\right)^{2}, \\
P & =\Delta F_{1}^{\prime \prime} \Delta F_{2}^{\prime}-\Delta F_{2}^{\prime \prime} \Delta F_{1}^{\prime} .
\end{aligned}
$$

Both $\sin \varphi_{0}(\mathbf{H})$ and $\cos \varphi_{0}(\mathbf{H})$ should be calculated in order to find the sign of the phase uniquely (Porai-Koshitz, 1968).

The essential obstacle for practical realization of the ASM on the basis of PXR is radiation damage of the investigated crystal by an incident electron beam. In the available experiments on PXR from non-relativistic electrons, thin singlecrystal films of thickness $L \sim 1 \div 10 \mu \mathrm{m}$ (Feranchuk et al., 2000) have been used, thus the limits for electron current density are the same as for transmission electron microscopy. If the ASM is assumed to be used for studies of organic objects like proteins, the probable radiation damage can be estimated in the following way. The expression (Landau \& Lifshitz, 1982) for energy loss by electrons of primary energy $E$ and current $J$ in unit time and within a layer of thickness $L$ is

$$
\Delta E \approx J \frac{4 \pi e^{4} Z_{\mathrm{tot}} L}{\Omega m c^{2}} B(E),
$$

where notations from (6) are used and $B(E)$ is a dimensionless function weakly depending on $E$. The energy change $\Delta E$ causes the temperature increase $\Delta T$ :

$$
k_{\mathrm{B}} \frac{\zeta_{\mathrm{tot}} S L}{\Omega} \Delta T \approx \Delta E .
$$

Here $k_{\mathrm{B}}$ is a Boltzmann constant, $k_{\mathrm{B}} \zeta_{\text {tot }}$ is the specific heat of the crystallographic unit cell, and $S$ is an electron beam cross section. From equations (44) and (45), the rough estimate for crystal temperature increase in unit time can be derived:

$$
\Delta T\left[\mathrm{~K} \mathrm{~s}^{-1}\right] \approx \frac{Z_{\mathrm{eff}}}{\zeta_{\mathrm{eff}}} \frac{J[\mu \mathrm{A}]}{S\left[\mathrm{~mm}^{2}\right]},
$$

where averaged values $Z_{\text {eff }}, \zeta_{\text {eff }}$ are normalized to a single atom of the crystallographic unit cell. The expression (46) can be used for the prediction of radiation damage of investigated samples. The practical values of this estimate have to be evaluated particularly for every possible ASM application.

\section{Conclusions}

The results of the present paper show that a detailed analysis of PXR spectra provides additional possibilities, in comparison with conventional X-ray diffraction. This advantage is very important when the structure of a complex organic crystal has to be solved. For organic crystals, parametric X-radiation combined with the ASM permits the phases of the structure amplitudes to be measured directly. The main purpose of the theoretical analysis considered here is to attract attention to a new field of PXR application and to initiate experimental studies on this radiation in organic crystals. The considered approach could be of great interest for practical X-ray structure analysis.

The authors thank the International Scientific Technical Center (Grant No. B-626) and Bruker AXS for support of this work. We are also grateful to A. A. Minkewich for help with the numerical calculations. 


\section{References}

Attfield, J. P. (1990). Nature (London), 343, 46-53.

Authier, A. (2001). Dynamical Theory of X-ray Diffraction. Oxford University Press/IUCr.

Backe, H., Kube, G. \& Lauth, W. (2001). Electron-Photon Interaction in Dense Media, edited by H. Wiellmann, pp. 153-189. Dordrecht: Kluwer Academic Publishers.

Baryshevsky, V. G., Danilov, V. A., Feranchuk, I. D. \& Shadyro, L. L. (1983). USSR patent 1032376 (priority 01.04.1983).

Baryshevsky, V. G. \& Feranchuk, I. D. (1972). Sov. Phys. JETP, 34, 502-506.

Baryshevsky, V. G. \& Feranchuk, I. D. (1983). J. Phys. (Paris), 44, 913-921.

Bijvoet, J. M. (1954). Nature (London), 173, 888-893.

Blazhevich, S. V., Bochek, G. I., Gavrikov, V. B., Kulibaba, V. I., Maslov, N. I., Nasonov, N. N., Pirogov, B. H., Safronov, A. G. \& Torgovkin, A. V. (1994). Phys. Lett. A195, 210-214.

Brenzinger, K.-H., Limburg, B., Backe, H., Dambach, S., Enteneuer, H., Hagenbuck, F., Herberg, C., Kaiser, K. H., Kettig, O., Kube, G., Lauth, W., Schöpe, H. \& Walcher, Th. (1997). Phys. Rev. Lett. 79, 2462-2465.

Chang, S. L. (1984). Multiple Diffraction of X-rays in Crystals. Heidelberg: Springer-Verlag.

Colella, R. (1974). Acta Cryst. A30, 413-419.

Feranchuk, I. D. (1979). Kristallografiya, 24, 289-296.

Feranchuk, I. D., Gurskii, L. I., Komarov, L. I., Lugovskaya, O. M., Burgäzy, F. \& Ulyanenkov, A. P. (2002). Acta Cryst. A58, 370-384.

Feranchuk, I. D. \& Ivashin, A. V. (1985). J. Phys. (Paris), 46, 1981-1986.

Feranchuk, I. D. \& Ivashin, A. V. (1989). Kristallografiya, 34, 39-46.

Feranchuk, I. D. \& Ulyanenkov, A. P. (1999). Acta Cryst. A55, 466-470.

Feranchuk, I. D. \& Ulyanenkov, A. P. (2001a). Phys. Rev. B, 63, 155318-1-7.

Feranchuk, I. D. \& Ulyanenkov, A. P. (2001b). Acta Cryst. A57, 283-289.

Feranchuk, I. D., Ulyanenkov, A. P., Harada, J. \& Spence, J. C. H. (2000). Phys. Rev. E, 62, 4225-4235.
Garibyan, G. M. \& Yang, C. (1972). Sov. Phys. JETP, 34, 495-502.

Gurskaya, G. V., Zavodnik, V. E. \& Shutalev, A. D. (2003). Kristallografiya, 48, 461-466.

Harker, D. (1956). Acta Cryst. 9, 1-7.

Hart, M. (1990). Nucl. Instrum. Methods, A297, 306-311.

Hart, M. \& Lang, A. R. (1961). Phys. Rev. Lett. 7, 120-122.

Hendrickson, W. A. (1991). Science, 254, 51-56.

James, R. W. (1960). The Optical Principles of the Diffraction of $X$-rays. London: G. Bell and Sons.

Kaplin, V. V., Uglov, S. R., Bulaev, O. F., Goncharov, V. G., Voronin, A. A., Piestrup, M. A., Gary, S. K., Nasonov, N. N. \& Fuler, M. K. (2002). Appl. Phys. Lett. 80, 3427-3431.

Krause, M. O. (1979). J. Phys. Chem. Ref. Data, 8, 307-308.

Krause, M. O. \& Oliver, J. H. (1979). J. Phys. Chem. Ref. Data, 8, 329-330.

Landau, L. D. \& Lifshitz, E. M. (1982). Electrodynamics of Condensed Media. Moscow: Nauka.

Mirkin, L. I. (1961). Handbook for X-ray Structure Analysis of Polycrystals. Moscow: Fizmatgiz.

Morokhovsky, V. V., Freudenberg, J., Genz, H., Morokhovskii, V. L., Richter, A. \& Sellschop, J. P. F. (2000). Phys. Rev. B, 61, 3347-3355.

Porai-Koshitz, M. A. (1968). Practical Course for X-ray Structure Analysis, Vol. 2. Moscow: Fizmatgiz.

Post, B. (1977). Phys. Rev. Lett. 39, 760-763.

Rullhansen, R., Artru, X. \& Dhez, P. (1998). Novel Radiation Sources Using Relativistic Electrons. Singapore: World Scientific.

Shen, Q. \& Colella, R. (1987). Nature (London), 329, 232-236.

Shen, Q. \& Huang, X.-R. (2001). Phys. Rev. B, 63, 174102-1-6.

Stetsko, Y. P., Lin, G.-Y., Huang, Y.-S. \& Chang, S.-L. (2001). Phys. Rev. Lett. 86, 2026-2030.

Ter-Mikaelyan, M. L.(1972). High Energy Electromagnetic Processes in Condensed Media, pp. 332-336. New York: Wiley.

Vainshtein, B. K. (1978). Modern Crystallography, Vol. 1. Moscow: Nauka.

Weckert, E. \& Hümmer, K. (1997). Acta Cryst. A53, 108-114.

Woolfson, M. M. \& Fan, H. F. (1995). Physical and Non-physical Methods of Solving Crystal Structures. Cambridge University Press.

Yeh, J. J. \& Lindau, I. (1985). At. Data Nucl. Data Tables, 32, $1-135$. 